\title{
The Role of a Single Formin Isoform in the Limb and Renal Phenotypes of Limb Deformity
}

\author{
Anthony Wynshaw-Boris,", Gabriella Ryan, ${ }^{\dagger}$ Chu-Xia Deng, \\ David C. Chan, ${ }^{*}$ Laurie Jackson-Grusby, ${ }^{*}$ Denise Larson, ${ }^{\dagger}$ \\ Judy H. Dunmore," and Philip Leder* \\ *Howard Hughes Medical Institute, Department of Genetics, Harvard \\ Medical School, Boston, Massachusetts, U.S.A. ${ }^{\dagger}$ Laboratory of \\ Genetic Disease Research, National Human Genome Research \\ Institute, National Institutes of Health, Bethesda, Maryland, U.S.A.
}

\begin{abstract}
Background: Mutations of the murine limb deformity (ld) locus are responsible for a pleiotropic phenotype of completely penetrant limb malformations and incompletely penetrant renal agenesis and/or dysgenesis. The ld locus encodes a complex family of mRNA and protein isoforms.

Materials and Methods: To examine the role of one of the more prominent of these isoforms, isoform IV, we specifically eliminated it by gene targeting.

Results: Unlike other mutant ld mice, homozygous mice bearing this isoform IV disruption display incompletely penetrant renal agenesis, but have perfectly normal limbs. Whole mount in situ hybridization demonstrated that this targeted disruption was specific for isoform IV and did not interfere with the expression of other $l d$ isoforms. The isoform IV-disrupted allele of $l d$
\end{abstract}

does not complement the renal agenesis phenotype of other ld alleles, in a manner consistent with its penetrance, and like the isoform IV-deficient mice, these compound heterozygotes have normal limbs. Sequence analysis of formin isoform IV in other $l d$ mutant alleles did not detect any amino acid changes relative to the strain of origin of the mutant allele.

Conclusions: Thus, the disruption of isoform IV is sufficient for the renal agenesis phenotype, but not the limb phenotype of $l d$ mutant mice. Structural mutations in this isoform are only one of several genetic mechanisms leading to the renal phenotype, since amino acid changes in this isoform were not detected. These results demonstrate that this gene is limb deformity, and that variable isoform expression may play a role in generating the pleiotropic ld phenotype.

\section{INTRODUCTION}

A number of murine and human complex dysmorphic syndromes are inherited in a Mendelian fashion $(1,2)$, suggesting the action of a single

Chu-Xia Deng's present address is Laboratory of Biochemistry and Metabolism, National Institute of Diabetes, Digestive and Kidney Diseases, National Institutes of Health, Bethesda, MD 20892, U.S.A.

Laurie Jackson-Grusby's present address is Whitehead Institute, Massachusetts Institute of Technology, Cambridge, Massachusetts, U.S.A.

Judy H. Dunmore's present address is Millenium Pharmaceuticals, Inc., Cambridge, Massachusetts, U.S.A.

Address correspondence and reprint requests to: Anthony Wynshaw-Boris, Laboratory of Genetic Disease Research, National Human Genome Research Institute, Bldg. 49/ 4A68, National Institutes of Health, Bethesda, MD 20892, U.S.A. Tel: 301-402-2034; Fax: 301-402-2170; e-mail: tonywb@nchgr.nih.gov. gene. While the pleiotropic nature of such singlegene disorders may result from multiple factors, tissue-specific control of gene expression is one likely mechanism for pleiotropy. For example, the activity of a single-gene product can be regulated in a tissue-specific manner at the level of transcription or translation. In addition, multiple gene products can be variably produced in different tissues by alternative promoter utilization and/or differential splicing, resulting in the localized expression of distinct proteins.

The mouse limb deformity locus (ld) provides a good model for the study of factors involved in the generation of complex dysmorphic syndromes from single genes. Five recessive mutants $\left(l d^{J}, l d^{O R}, l d^{T g H d}, l d^{T g B r i}\right.$, and $\left.l d^{l n 2}\right)$ of this locus have previously been described (3-9). Homozy- 
gous mutant mice have oligosyndactyly with fusion of the long bones of the limbs, as well as renal agenesis, hypoplasia, and dysplasia. The limb phenotype is completely penetrant in homozygotes, but the renal agenesis phenotype shows incomplete penetrance and variable expressivity. Each of the five known alleles have differences in penetrance of the renal phenotype, which vary from $98 \%$ in $l d^{J} / l d^{J}$ mice (10) to $21 \%$ in $l d^{\ln 2} / l d^{\ln 2}$ mice (9). These differences may be a consequence of the strength of each allele and/or strain background differences (10).

The gene for the ld locus was cloned via a transgene insertion in the $l d^{\text {TgHd }}$ allele (7). The gene is composed of at least 24 exons spanning over $400 \mathrm{~kb}$ (11a), and has at least four different mRNA isoforms that are differentially expressed during development (see Fig. 1A) (11). Isoforms I-III contain a common $5^{\prime}$ exon that codes for a basic protein domain, while isoform IV contains a unique $5^{\prime}$ terminal exon encoding an acidic domain. Isoforms I-III are coordinately expressed in the developing kidney and central and peripheral nervous systems, but they are expressed only at very low levels in the limb buds. Isoform IV is expressed at high levels in the limb buds, particularly the apical ectodermal ridge (AER), developing kidneys, notochord, branchial arches, somites, and tail buds, as determined by RNase protection (11) and in situ hybridization analysis (12). In the chicken, a similar pattern of expression was seen using antibodies to the isoform IV product (13). In addition, there are at least two distinct promoters in the ld locus: one at the $5^{\prime}$ end of isoforms I-III and an internal promoter for isoform IV. The existence of the internal promoter was demonstrated using a lacZ reporter assay in transgenic mice (12). These observations suggest distinct roles for isoforms I-III and IV in the ld phenotype.

Given that $l d$ is a complex locus giving rise to multiple protein isoforms, we were interested in determining whether components of the pleiotropic $l d$ phenotype might be the consequence of a mutation affecting one of these isoforms. To test this, we have investigated the role of isoform IV in the ld phenotype by isoform-specific gene targeting, and by sequence analysis of this isoform in spontaneously occurring $l d$ mutants. Our results indicate that the disruption of isoform IV results in the occurrence of one of the mutant $l d$ phenotypes, renal agenesis/dysgenesis phenotype, but not the limb phenotype that accompanies other mutations at the $l d$ locus.
A
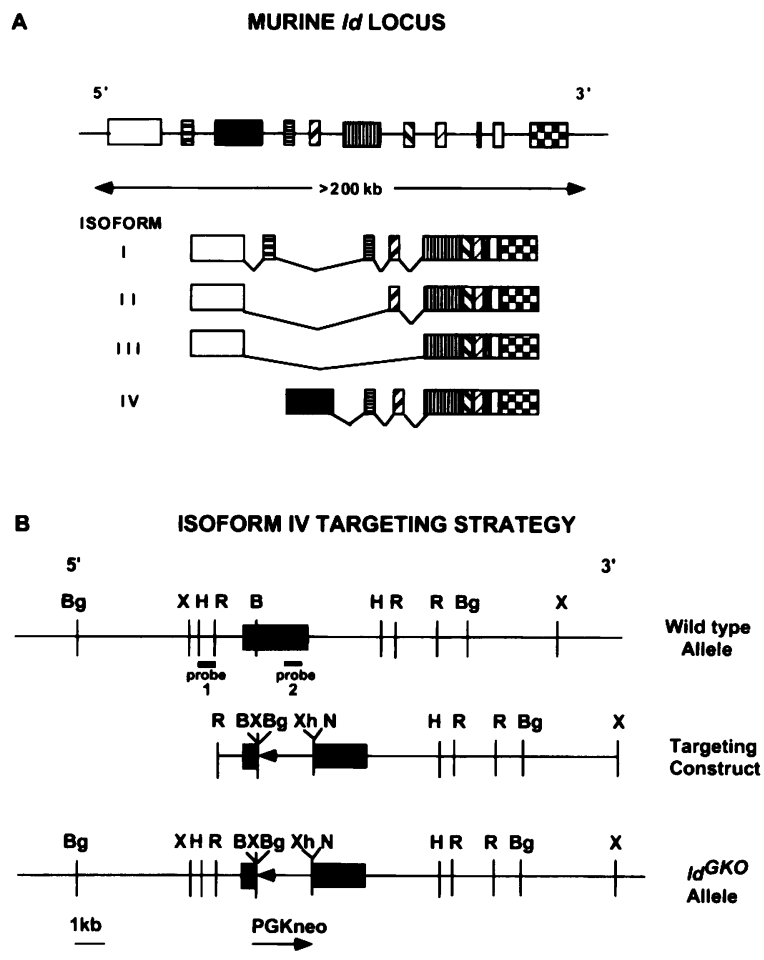

FIG. 1. Disruption of isoform IV of the $l d$ gene (A) Murine $l d$ locus. The alternative splicing of isoforms I-IV are shown (after Jackson-Grusby et al. 1992). Not all 24 exons are shown, only those important for distinguishing the four isoforms. (B) Targeting strategy. Top: genomic organization of the wild-type $l d$ gene (129 strain) around the $5^{\prime}$-terminal exon (black box) of isoform IV. Middle: replacement-type targeting construct for isoform IV. A PGK-neo gene (arrow, indicating direction of transcription) was inserted into this exon at a unique BamHI site of isoform IV, in the opposite orientation relative to transcription, and introduced stop codons in all three reading frames of isoform IV. Bottom: structure of the mutant locus ( $\left(d^{G K O}\right.$ allele) after homologous recombination with the targeting construct. The location of the flanking (probe 1) and internal (probe 2) probes used for Southern analysis are indicated by bars under the wild type allele. Restriction enzyme sites: B, BamHI; $\mathrm{Bg}, \mathrm{BgIII} ; \mathrm{H}$, HindIII; N, NotI; R, EcoRI; X, XbaI; Xh, XhoI.

\section{MATERIALS AND METHODS}

\section{Gene Targeting}

The 129Sv genomic clone for the 5 '-terminal exon of isoform IV was isolated from a $129 \mathrm{~Sv}$ female liver genomic library (Stratagene). The targeting construct was made in the vector pPNT (14), and Jl ES cells (15) were used for transfection. The growth, transfection, and isolation of potential targeted clones were carried out as pre- 
viously described (16). One hundred ninety-six clones were analyzed for homologous recombination by nested polymerase chain reaction (PCR) (data not shown), and by Southern analysis using a flanking primer and a neo primer. Eight PCR positive clones were expanded and correct targeting in six clones was confirmed by Southern blot analysis with five different restriction enzymes, using either a flanking probe (Fig. 1B, probe 1) or an internal probe (Fig. 1B, probe 2) from the exon. After blastocyst injection, one targeted line gave germ-line transmission. The production of chimeric animals and mating of chimeric males with NIH Black Swiss (Taconic) females to test for germ-line chimerism has been described elsewhere (16). F1 heterozygotes were intercrossed to generate all genotypes, and the line was maintained in this mixed background.

\section{Skeletal Stains}

Skeletal stains were done as described previously (17). The axial skeletons of $l d^{G K O} / l d^{G K O}$ animals were normal (data not shown), as previously reported for all other $l d$ alleles $(3-9,18,19)$.

\section{Whole Mount in Situ Hybridization Analysis}

Experiments were performed as described previously (20), with the exception that embryos were permeablized with detergents (21), as opposed to proteinase $\mathrm{K}$ treatment when examining AER staining patterns (12). Probes for isoforms I-III and isoform IV were carried out as described elsewhere (12).

\section{Immunoblot Analysis}

For immunoblot analysis, protein samples were separated on $6 \%$ acrylamide Laemmli gels, transferred to Immobilon-P (Millipore), and hybridized with the appropriate antiserum. Adult animals were genotyped by Southern analysis of tail DNA, while embryos were the result of homozygous matings, and therefore of uniform genotype. An entire litter (8-9 embryos) was dissected for limb bud samples, while only two heads or two bodies (devoid of head and limb buds) were used for those samples. After dissection, the embryo samples were quickly frozen at $-80^{\circ} \mathrm{C}$. Samples were disrupted by Dounce homogenization in PBS with protease inhibitors. One-half of each sample was loaded in each well.
Each genotype from each embryonic fraction was repeated at least twice, with similar results (data not shown). Polyacrylamide gel electrophoresis, immunoblotting, and stripping of filters were performed as described elsewhere (22).

\section{Sequence Analysis}

cDNA representing the entirety of the coding sequence of isoform IV were synthesized from homozygous kidney samples from FVB, $l d^{J}$ and $l d^{O R}$ mice, using SuperScript (GIBCO/BRL). Five primer pairs were used to amplify overlapping 800- to 1,000-bp fragments from isoform IV (sequences and PCR conditions available upon request). PCR products were cloned by the T-vector kit (InVitrogen), and two clones of each fragment were sequenced on one strand each to give complete coverage for isoform IV. Automated sequencing was performed on an Applied Biosystems, Inc., machine, using conditions recommended by the manufacturer. Sequence polymorphisms were confirmed by repeat sequencing of both strands of the same fragment. If confirmed, the relevant region of isoform IV was directly amplified from genomic DNA. The PCR fragments were directly sequenced, or the presence of polymorphic restriction enzyme sites from the polymorphic nucleotides were determined.

\section{RESULTS}

\section{Targeted Disruption of Isoform IV}

Unique $5^{\prime}$ exons are used for isoforms I-III and isoform IV (Fig. 1A). A replacement-type targeting construct was designed to disrupt isoform IV (Fig. 1B), using a 129-Sv genomic clone of the $l d$ locus surrounding the $5^{\prime}$-terminal exon of isoform IV for the targeting construct. This exon is adjacent to the isoform IV promoter and is not utilized in isoforms I-III (Fig. 1A). A PGK-neo cassette was inserted $195 \mathrm{bp} \mathrm{3'}$ of the start codon (65 amino acids into the open reading frame) of this exon, in the opposite orientation relative to transcription. Stop codons were introduced in all three reading frames. This insertion into the $5^{\prime}$ terminal acidic exon of isoform IV is predicted to result in either a severely truncated, nonfunctional protein from the isoform IV transcript, or no protein product, and should have no effect on the expression of isoforms I-III.

This construct was introduced into $\mathrm{Jl}$ em- 
bryonic stem cells (15), and nine clones containing the disrupted allele $\left(l d^{G K O}\right)$ were identified using a nested PCR strategy, with neo primers and primers from the flanking region $5^{\prime}$ of the targeting construct (data not shown). These nine positive clones were analyzed by Southern blotting, using a flanking probe (probe 1, Fig. 1B) or an internal probe (probe 2, Fig. 1B). After Xbal digestion, the predicted polymorphic band of 2 $\mathrm{kb}$ and the wild-type $12-\mathrm{kb}$ band were observed in eight clones using probe 1 (Fig. 2A), demonstrating appropriate targeting. Using probe 2 after HindIII digestion, correct targeting was confirmed by the presence of a polymorphic $8-\mathrm{kb}$ band in seven clones, in addition to the wild-type $6-\mathrm{kb}$ band (Fig. 2B). Using several different enzyme digestions and these probes, five of the clones had completely correct targeting (data not shown). However, the other PCR-positive clones had bands of inappropriate sizes (see Fig. 2A, lane 2 and Fig. 2B, lanes 2 and 5), suggesting that targeting was aberrant in these clones. One of three correctly targeted clones resulted in germline transmission after injection into blastocysts, establishing the $l d^{G K O}$ isoform IV mutation in mice.

\section{Immunoblot Analysis of Isoform IV in Mutant Animals}

Fl $l d^{G K O} /+$ heterozygotes were crossed, and all possible ld genotypes were observed in the offspring (Fig. 2C). Over 100 offspring resulting from heterozygous crosses were genotyped, and all genotypes were present in the expected 1:2:1 $\left(+1+: l d^{G K O} /+: l d^{G K O} / l d^{G K O}\right)$ ratio. To determine if the disruption resulted in total loss of isoform IV protein, we performed Western blot analysis of embryos that resulted from heterozygous crosses. The different isoforms of the ld locus potentially encode a family of proteins, termed the formins (23). In vitro translation studies demonstrated that isoforms I, II, and IV encode proteins of 180,160 , and $165 \mathrm{kDa}$, respectively, and isoform III encodes a protein of $78 \mathrm{kDa}(24)$. To examine the expression of formin isoform IV, we used a rabbit antiserum that was made to the $5^{\prime}$ end of the proline-rich exon of the mouse protein ( $\alpha$-proline antiserum). This exon is common to all four isoforms; however, this antiserum only detects a $165-\mathrm{kDa}$ protein corresponding to isoform IV in embryonic samples (see below).

Immunoblots of kidney lysates from wildtype animals, using the $\alpha$-proline antiserum,
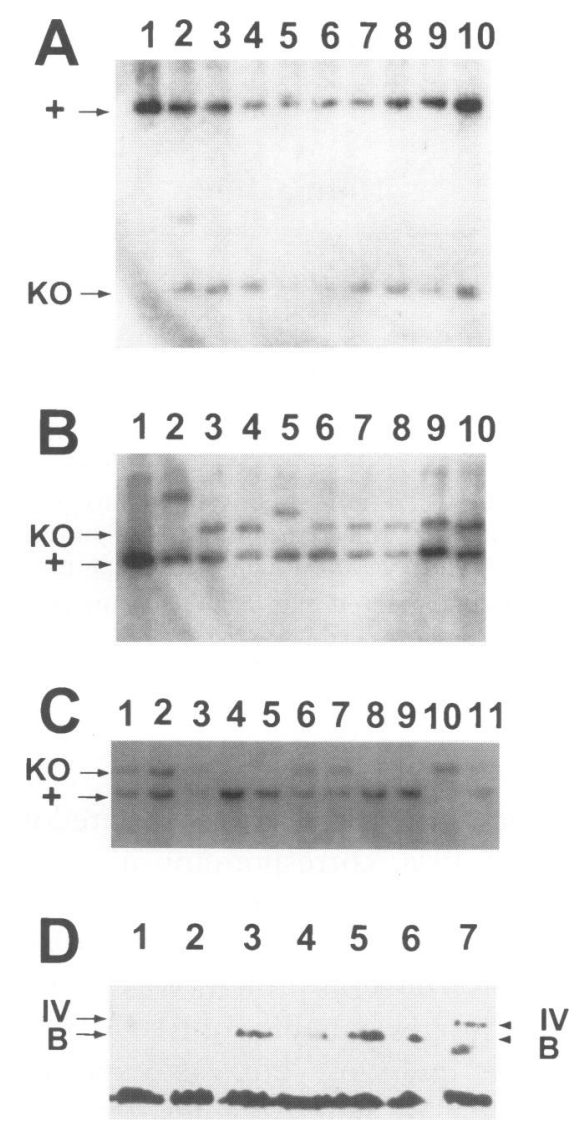

FIG. 2. Southern and immunoblot blot analysis of ES clones and animals

(A) Southern analysis of DNA from targeted ES clones, after $\mathrm{XbaI}$ digestion, and using a flanking probe (probe 1 from Fig. 1B). Lane 1, control J1 ES cell DNA; lanes 2-10, PCR-positive clones; + , wildtype allele $(12 \mathrm{~kb}) ; \mathrm{KO}, l d^{G K O}$ allele $(2 \mathrm{~kb})$. (B) Southern analysis of the same ES clones, after HindIII digestion, using an internal probe (probe 2 from Fig. 1B). +, wild-type allele $(6 \mathrm{~kb}) ; \mathrm{KO}, l d^{G K O}$ allele $(8 \mathrm{~kb})$. (C) Southern analysis of mice containing the $l d^{G K O}$ allele. Tail DNA samples from the F2 offspring of a heterozygous cross (lanes 1-11) were analyzed by Southern blot analysis after EcoRI digestion, using the internal probe 2 . + , wild-type allele $(6 \mathrm{~kb}) ; \mathrm{KO}, l d^{G K O}$ allele $(8 \mathrm{~kb})$. Pups $4,5,8,9$, and 11 were $+/+$, pups $1-3,6$, and 7 were $l d^{G K O} /+$, and pup 10 was $l d^{G K O} / l d^{G K O}$. (D) Immunoblot analysis of lysates from bodies, dissected away from head and limb buds, of E10.5 embryos of different genotypes. The genotypes analyzed were: $l d^{G K O} / l d^{G K O}$ homozygotes (lanes 1,2$) ; l d^{G K O} / l d^{T g B r i}$ compound heterozygotes (lanes 3-6); and $l d^{G K O} /+$ heterozygote (lane $7)$. The relevant protein bands are indicated by arrows: IV refers to the $165-\mathrm{kDa}$ isoform IV protein; $\mathrm{B}$ refers to the $155-\mathrm{kDa} l d^{T g B r i}$ truncated protein. A background band of about $120 \mathrm{kDa}$ was shown to demonstrate relative protein loading in each lane. 
TABLE 1. Limb and Kidney Phenotypes of F1 Compound Heterozygotes from Homozygous Crosses

\begin{tabular}{|c|c|c|c|c|c|c|}
\hline \multirow[b]{2}{*}{ Genotype } & \multirow[b]{2}{*}{ Normal limbs } & \multicolumn{3}{|c|}{ Normal kidneys } & \multirow{2}{*}{$\begin{array}{c}\text { Abnormal } \\
(\%)\end{array}$} & \multirow{2}{*}{$\begin{array}{c}\text { Kidney } \\
\text { abnormality }\end{array}$} \\
\hline & & $\mathbf{0}$ & $\mathbf{1}$ & 2 & & \\
\hline$l d^{G K O} /+$ & $92 / 92$ & 0 & 0 & 92 & 0 & None \\
\hline$l d^{G K O} / l d^{G K O}$ & $105 / 105$ & 1 & 5 & 99 & $6 \% *$ & Agenesis \\
\hline$l d^{G K O} / l d^{T g B r i}$ & $62 / 62$ & 0 & 1 & 61 & $2 \%$ & Agenesis \\
\hline$l d^{G K O} / l d^{\ln 2}$ & $43 / 43$ & 1 & 0 & 42 & $2 \%$ & Multicystic dysplastic \\
\hline
\end{tabular}

Matings were between homozygous parents to give uniform genotypes in each litter. Pregnant females were checked twice daily for litters to minimize the chance of missing pups that died at birth. All pups were scored for limb phenotype and for the number of kidneys by inspection under a dissecting microscope.

${ }^{*} p<0.05$, when compared with $l d^{G K O} /+$ neonates by Fisher's exact test.

demonstrated the presence of a predominant band of $165 \mathrm{kDa}$, corresponding in size to isoform IV, which was undetectable in lysates of $l d^{G K O} / l d^{G K O}$ homozygote kidneys (data not shown). This $165-\mathrm{kDa}$ protein was also missing from crude lysates of bodies of E10.5 $l d^{G K O} / l d^{G K O}$ homozygote embryos, but, as expected, was present in $l d^{G K O} /+$ heterozygotes (Fig. 2D). Similar results were obtained using an antiserum raised against the $5^{\prime}$-terminal exon of isoform IV (data not shown), demonstrating that this protein represents the translation product of isoform IV and that it is absent in the null embryos. The $l d^{T g B r i}$ allele, resulting from a transgene insertion $(8,19)$, codes for a truncated $(155 \mathrm{kDa})$ protein of isoform IV (D.C. Chan, unpublished observations). Body lysates from $l d^{G K O} / l d^{T g B r i}$ compound heterozygotes contained only this truncated protein, and did not contain detectable levels of the 165-kDa protein (Fig. 2D, lanes 3-6), providing additional evidence that the isoform IV disrupted allele does not make any of the normal isoform IV $165-\mathrm{kDa}$ protein. Identical results were obtained from immunoblot analysis of dissected limb buds and head samples of E10.5 embryos (data not shown). These results demonstrate that no isoform IV protein was produced from the $l d^{G K O}$ allele and confirm that we have eliminated this isoform in these mice.

\section{Limb and Kidney Phenotypes of Mutant Mice}

All $l d^{G K O} / l d^{G K O}$ animals appeared grossly normal by inspection and were healthy and fertile. To assess the phenotype of $l d^{G K O} / l d^{G K O}$ homozygotes and compound heterozygotes formed between $l d^{G K O}$ and other $l d$ mutant alleles, $l d^{G K O} / l d^{G K O}$ homozygous males were mated to $+1+, l d^{G K O} /$ $l d^{G K O}, l d^{T g B r i} / l d^{T g B r i}$, and $l d^{l n 2} / l d^{l n 2}$ homozygous females, and neonates were scored for limb and kidney abnormalities. All heterozygous $l d^{G K O} /+$ and homozygous $l d^{G K O} / l d^{G K O}$ neonates had normal limbs by inspection (Table 1) and skeletal staining (Fig. 3A,B). In particular, all animals had normal numbers of digits, absence of syndactyly, and absence of radioulnar and tibiofibular synostosis, which are the hallmarks of the $l d$ limb phenotype. $l d^{G K O} / l d^{T g B r i}$ and $l d^{G K O} / l d^{\ln 2}$ compound heterozygotes also had entirely normal limbs (Table 1). These findings demonstrate that the disruption of isoform IV is not sufficient for the limb phenotype of the ld locus and that the $l d^{G K O}$ mutant allele complements the limb phenotype of other $l d$ alleles.

In contrast to the absent limb phenotype, $6 \%(6 / 105)$ of $l d^{G K O} / l d^{G K O}$ homozygous mutants had unilateral or bilateral renal agenesis (Table 1 , Fig. 3C,D). The adrenal glands were always present bilaterally. In some cases, blind-ended ureters originating from the bladder were present, while in others, the ureters were absent on the ipsilateral side (Fig. 3C,D). Of $62 \mathrm{ld}^{G K O} /$ $l d^{T g B r i}$ compound heterozygote neonates, one animal $(2 \%)$ had unilateral agenesis (Table 1). Also, one $l d^{G K O} / l d^{l n 2}$ compound heterozygote neonate had bilateral multicystic dysplastic kidneys (Table 1, Fig. 3E-G), a phenotype within the spectrum of renal agenesis and seen previously in $l d^{l n 2} / l d^{l n 2}$ mutants $(9,10)$. The tubules of such kidneys are characterized by developmental malformations, resulting in huge urine-filled cysts 

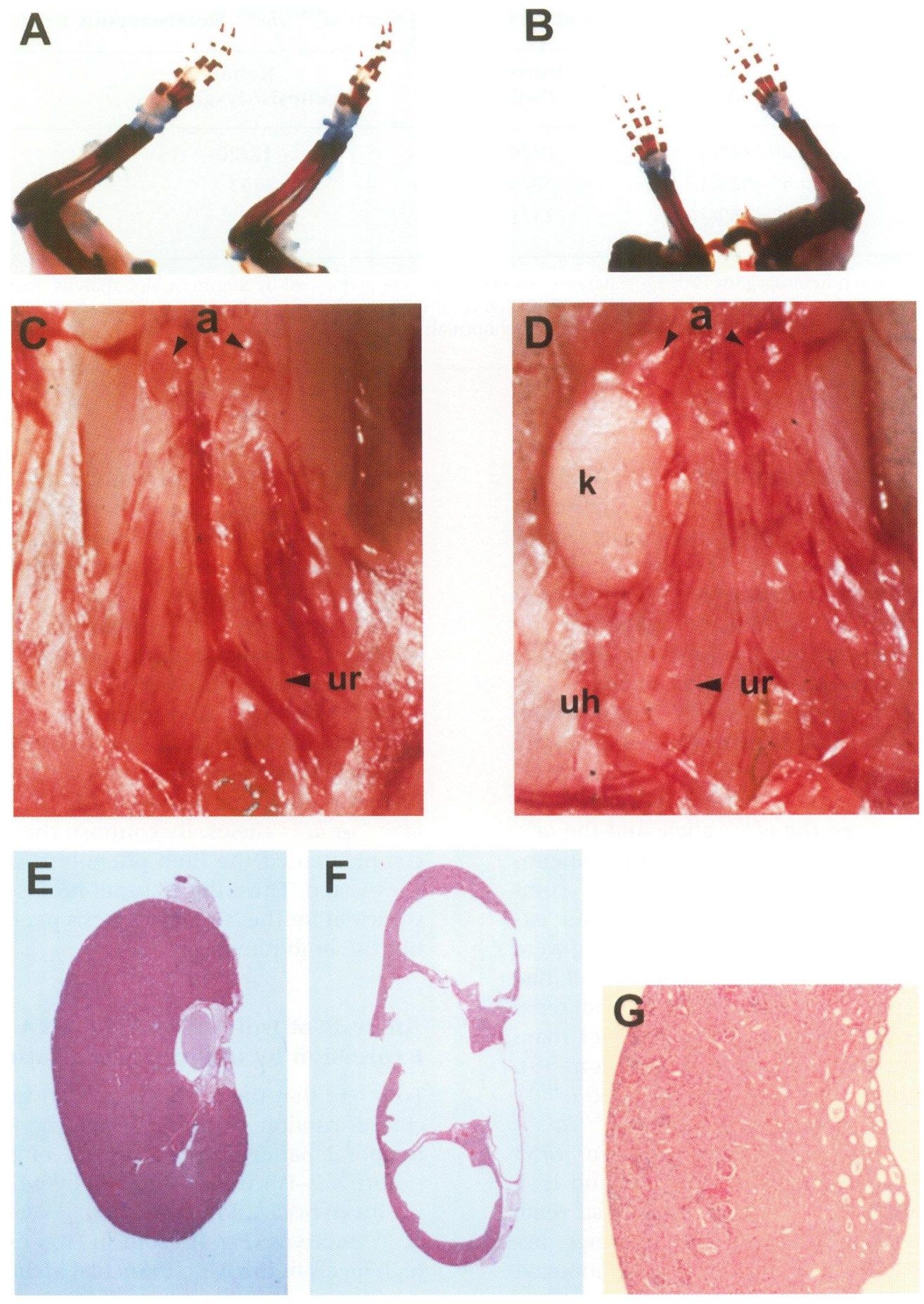

FIG. 3. Analysis of mutant mice

(A) and (B) Forelimbs after skeletal staining. Note that both limbs have the normal number of digits, and both have paired long bones (radius and ulna). Left, $l d^{G K O} /+\mathrm{limb}$; right, $l d^{G K O} / l d^{G K O}$ limb. (C) $l d^{G K O} / l d^{G K O}$ animal with bilateral renal agenesis. The adrenal glands (a) were present bilaterally, but the ureter (ur) was present only on the left side. (D) $l d^{G K O} / l d^{G K O}$ animal with unilateral (left) renal agenesis. The adrenal glands (a) were present bilaterally, but the ureter (ur) was present only on the side with the kidney $(\mathrm{k})$ and distinguished from the uterine horn (uh) on the same side. (E) Kidney from a 2-week-old $+/+$ mouse, sectioned and stained with hematoxylin and eosin. (F) Kidney from a $l d^{G K O} / l d^{l n 2} 2$-week-old littermate with bilateral multicystic dysplastic kidneys, sectioned and stained with hematoxylin and eosin. Note the massive cysts, due to maldevelopment of the excretory system. (G) Part of kidney section in F (higher magnification). The small cysts on the right of the figure reflect the maldevelopment associated with the multicystic dysplastic kidney phenotype. 
TABLE 2. Limb and Kidney Phenotypes of F2 Offspring from $l d^{G K o} / l d^{\ln 2}$ Heterozygous Crosses

\begin{tabular}{lcccc}
\hline Genotype & $\begin{array}{c}\text { Total } \\
(\%)\end{array}$ & $\begin{array}{c}\text { Normal } \\
\text { limbs }\end{array}$ & $\begin{array}{c}\text { Renal } \\
\text { agenesis/dysgenesis }\end{array}$ & $\begin{array}{c}\text { Abnormal } \\
\text { (\%) }\end{array}$ \\
\hline$l d^{\ln 2} / l d^{\ln 2}$ & $20(24 \%)$ & $0 / 20$ & $12 / 20$ & $60 \%$ \\
$l d^{G K O} / l d^{\ln 2}$ & $53(62 \%)$ & $53 / 53$ & $6 / 53$ & $11 \%$ \\
$l d^{G K O} / l d^{G K O}$ & $11(13 \%)$ & $11 / 11$ & $1 / 11$ & $9 \%$
\end{tabular}

Matings were between heterozygous $l d^{G K O} / l d^{l n 2}$ parents. All offspring were genotyped by Southern blot analysis. Pregnant females were checked twice daily for litters to minimize the chance of missing pups that died at birth. All pups were scored under a dissecting microscope for limb phenotype and for kidney abnormalities.

from defective tubulogenesis (Fig. 3F, compared with a normal kidney in Fig. 3E). At high magnification (Fig. 3G), even the normal-appearing parenchyma at the margin of this multicystic dysplastic kidney showed evidence of smaller cysts, reflecting the hindrance of urine flow resulting from tubular maldevelopment. We have not observed renal agenesis in 92 neonatal heterozygous $l d^{G K O} /+$ mice (Table 1 ), nor in several hundred wild-type animals in this mixed strain background (data not shown).

To examine in more detail the noncomplementation between the $l d^{G K O}$ allele and the $l d^{\ln 2}$ allele, we analyzed the limb and kidney phenotypes of F2 offspring of an F1 $l d^{G K O} / l d^{l n 2}$ compound heterozygous cross (Table 2). As expected, approximately $25 \%$ of the offspring were $l d^{\ln 2} / l d^{\ln 2}$ homozygotes. These animals all had abnormal limbs, and $60 \%$ of them had renal agenesis or dysgenesis, which was higher than, but consistent with, previous observations (9). The remaining neonates, which included $l d^{G K O}$, $l d^{\ln 2}$ compound heterozygotes and $l d^{G K O} / l d^{G K O}$ homozygotes, had normal limbs. However, 6/53 $(11 \%)$ of the compound heterozygotes and $1 / 11$ (9\%) of the $l d^{G K O} / l d^{G K O}$ homozygotes had renal agenesis/dysgenesis. In separate matings between wild-type mice and $l d^{\ln 2} / l d^{\ln 2}$ homozygotes, out of 42 offspring, none had renal agenesis/dysgenesis, demonstrating that the renal phenotype observed in these mice was caused only by the ld alleles. There was a relatively lower number of $l d^{G K O} / l d^{G K O}$ homozygotes $(13 \%)$. We have not yet determined whether this occurred by chance or was due to aneuploidy from segregation of the abnormal chromosomes in the $l d^{l n 2}$ mice (9). We have not observed any other evidence that the isoform IV disruption had an effect on embryonic viability.
In support of this interpretation, the litter sizes of matings between mice that were homozygous for the isoform IV disruption averaged between 9 and 10 mice, which is similar to wild-type litter sizes (data not shown).

These results indicate that the disruption of isoform IV results in renal agenesis/dysgenesis, with penetrance somewhat lower than that observed with other ld mutant alleles. Consistent with this observation, a low incidence of noncomplementarity of the renal agenesis phenotype was observed between the $l d^{G K O}$ and the $l d^{T g B r i}$ or $l d^{l n 2}$ alleles. By contrast, the $l d^{G K O}$ allele complemented the limb phenotype of all $l d$ alleles tested. Thus, the disruption of isoform IV is sufficient for the ld renal agenesis phenotype, but not the limb phenotype.

\section{Analysis of Isoform-specific RNA Expression by in Situ Hybridization}

To prove that the $l d^{G K O}$ allele is an isoform-specific disruption of the $l d$ locus, the spatial and temporal patterns of expression of the formin isoforms (I-IV) were examined by whole-mount in situ hybridization analysis in $+/+$ and $l d^{G K O} /$ $l d^{G K O}$ embryos (Fig 4). Isoform IV is expressed at high levels in the AER, branchial arches, and in a punctate pattern in the somites of E9.5 and E10.5 embryos, but not in their dorsal root ganglia (DRG) or trigeminal ganglia (TG). In contrast, isoforms I-III are expressed in the DRG and TG of E10.5 embryos, but not the AER or somites (12). Therefore, the AER, somites, DRG and TG represent areas of unique expression of $l d$ isoforms. In addition, isoforms I-III and IV are coordinately expressed in the mesonephros, the ureteric bud, and the branching derivatives of the bud (12). 


\section{I-III}
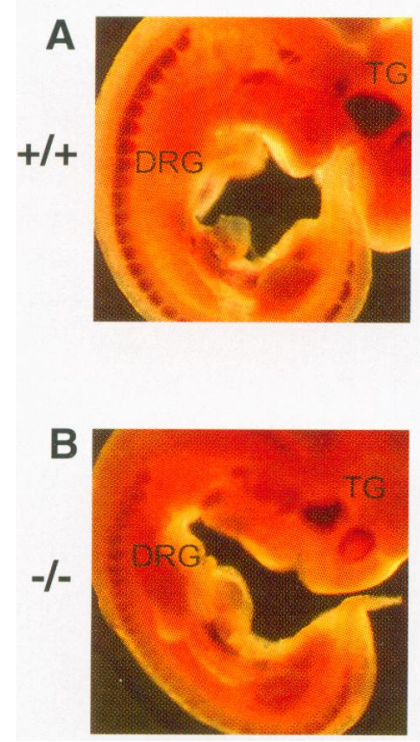
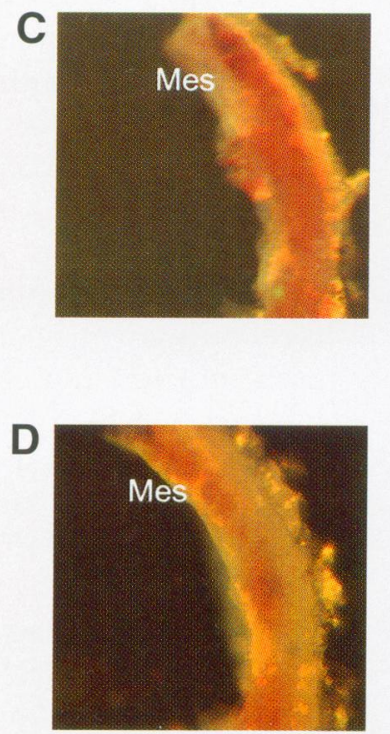
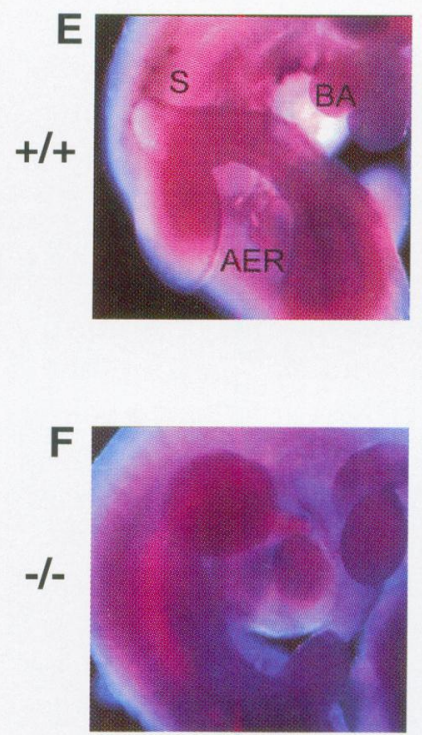

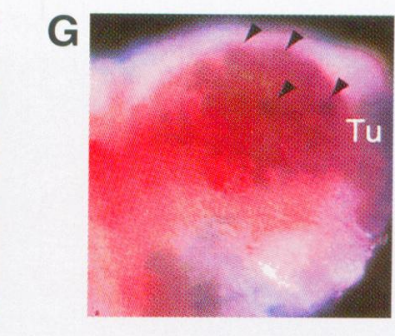

IV

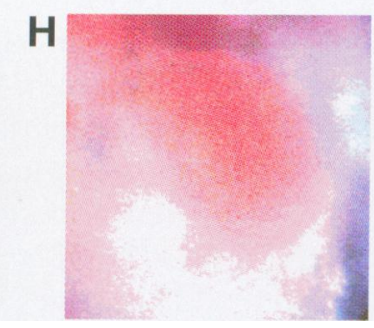

FIG. 4. Whole-mount in situ analysis of isoforms I-III and IV in wild-type and mutant mice $+1+$ (A) and $l d^{G K O} / l d^{G K O}(-1-)$ (B) El 1.5 embryos, and dissected mesonephroi from $+1+(\mathrm{C})$ and $l d^{G K O} / l d^{G K O}$ $(-/-)$ (D) El0.5 embryos were hybridized with an isoform I-III RNA antisense probe. $+/+\left(\right.$ E) and $l d^{G K O} / l d^{G K O}$ (F) E10.5 embryos, and dissected kidneys from $+l+(\mathrm{G})$ and $l d^{G K O} / l d^{G K O}(\mathrm{H})$ El 3.5 embryos were hybridized with an isoform IV RNA antisense probe. AER, apical ectodermal ridge; S, punctate staining in the somites; DRG, dorsal root ganglia; TG, trigeminal ganglia; Mes, mesonephric tubules; Tu, metanephric tubules (arrowheads); BA, branchial arches.

Using an isoform I-III-specific antisense RNA probe, the staining patterns of the DRG and TG were identical between E1 $1.5+/+$ (Fig. 4A) and $l d^{G K O} / l d^{G K O}$ embryos (Fig. 4B), as well as in E9.5 and E10.5 embryos (data not shown). In contrast, using an isoform IV-specific antisense RNA probe, there was intense staining of the AER, somites, and branchial arches of $+1+$ E9.5 (data not shown) and E10.5 embryos (Fig. 4E). However, this staining pattern was absent in $l d^{G K O} / l d^{G K O} \mathrm{E} 9.5$ (data not shown) or E10.5 embryos (Fig. 4F).

The pattern of expression of isoforms I-III and IV was examined in the developing kidneys. The expression of isoform I-III in the mesonephric ducts and tubules was identical between $+/+$ (Fig. 4C) and $l d^{G K O} / l d^{G K O}$ (Fig. 4D) embryos. In addition, the ureteric bud and branching derivatives were stained with a probe to isoforms I-III in embryos of both genotypes (data not shown). However, isoform IV was expressed in the developing kidneys only in $+1+$ embryos. Isoform IV was detectable in the mesonephric ducts and tubules, ureteric bud (data not shown), and in a punctate pattern from branching of the ureteric bud in older (E13.5) kidneys (Fig. 4G). In $l d^{G K O}$ / $l d^{G K O}$ embryos, no expression of isoform IV was detectable in mesonephroi, ureteric buds (data not shown), or E13.5 kidneys (Fig. 4H). Thus, the spatial and temporal expression of isoform IV RNA is specifically disrupted in the $l d^{G K O}$ allele, whereas the expression of RNA from isoforms I-III is unaffected.

\section{No Isoform IV Mutations in the $l d^{J}$ or $l d^{\text {oR }}$ Alleles}

The $l d^{T g B r i}$ and $l d^{l n 2}$ alleles are the result of a transgene insertion and a chromosomal translocation, respectively, at the $3^{\prime}$-end of the formin gene $(9,19)$, which disrupt all known isoforms (I-IV) of the ld locus. As expected, these disruptions result in the production of slightly truncated isoform IV proteins as assessed by immunoblot analysis (D. C. Chan and P. Leder, unpublished observations, and results presented above). In contrast, the molecular basis of the mutations responsible for the $l d^{J}$ and $l d^{O R}$ alleles 
have not been described. These spontaneously occurring alleles are likely to contain point mutations or small deletions or insertions, since no genomic rearrangements have been detected by Southern blot analysis (data not shown) and the proteins produced from these alleles are unaltered in size or quantity by immunoblot analysis (D. C. Chan and P. Leder, unpublished observations).

To determine whether mutations in isoform IV are present in the $l d^{J}$ and $l d^{O R}$ alleles, the sequences of isoform IV cDNAs for these alleles were determined and compared with the published sequence from FVB mice (11). Reverse transcription polymerase chain reaction (RTPCR) products encompassing the ORF of isoform IV were cloned and sequenced from wild-type (FVB), $l d^{O R} / l d^{O R}$ and $l d^{J} / l d^{J}$ kidney RNA samples. No nucleotide changes were detected in the coding sequence of isoform IV in the wild-type allele of FVB mice. No mutations or polymorphisms were found in the $l d^{O R}$ allele, either. However, we found four differences resulting in amino acid changes in isoform IV of the $l d^{J}$ allele: (1) a 3-bp deletion from 2304-2306, resulting in the deletion of a proline residue $(\Delta \mathrm{P} 694) ;(2)$ an $A \rightarrow G$ transition at position 1372 (Al372G), which results in an arg $\rightarrow$ gly amino acid substitution at amino acid 383 (R383G); (3) a G $\rightarrow$ A transition at position 1322 (Gl322A), which results in a gly $\rightarrow$ asp amino acid substitution at amino acid 366 (G366N); and (4) an A $\rightarrow$ G transition at position 3450 (A3450G), which results in a cys $\rightarrow$ trp amino acid substitution at amino acid 1076 (C1077W). To determine if these changes occur in the strain of origin of the $l d^{J}$ allele, the $\Delta \mathrm{P} 694$ region and the region surrounding amino acid 1077 were sequenced from CBA/Ca mice, which is the strain in which the $l d^{J}$ allele arose. The G1322A and Al372G mutations introduced novel restriction enzyme recognition sites. PCR fragments surrounding these sites were amplified and digested with the appropriate enzymes from $l d^{J} / l d^{J}$ and CBA/Ca DNA. All four mutations were present in $\mathrm{CBA} / \mathrm{Ca}$, indicating that they are strain polymorphisms. Since CBA/Ca mice have normal limbs and kidneys, these polymorphisms cannot be responsible for the abnormal function of the $l d^{J}$ allele. Therefore, there are no pathological isoform IV mutations in the $l d^{J}$ or $l d^{O R}$ alleles, demonstrating that isoform IV mutations are not necessary for the limb and kidney phenotypes of these spontaneous mutants of the $l d$ locus.

\section{DISCUSSION}

In an effort to dissect the molecular basis for individual components of the complex $l d$ phenotype, we have examined the role of an individual isoform of the $l d$ locus in the limb deformity phenotype. Isoform IV is expressed from an internal promoter (12) and is an independent transcriptional unit, distinct from isoforms I-III. By disrupting the $5^{\prime}$-terminal exon of isoform IV, we have created mice that lacked this isoform but retain normal expression of isoforms I-III. Mice that are selectively and completely deficient for isoform IV manifest incompletely penetrant renal agenesis, yet they have normal limbs. In addition, the isoform IV-disrupted allele does not complement the renal agenesis phenotype of two ld mutant alleles. Thus, loss of function of isoform IV is sufficient for the low incidence of the renal agenesis/dysgenesis phenotype of the $l d$ locus, but not the limb phenotype. However, whereas elimination of isoform IV is sufficient to produce the renal phenotype, mutations in this isoform are not necessary for either the limb or kidney phenotype of $l d$, since no isoform IV mutations were found in either the $l d^{J}$ or $l d^{O R}$ alleles, which produce both manifestations of the $l d$ phenotype. Our results uncover an important role for an individual isoform of the $l d$ locus in the generation of the complex limb deformity phenotype and suggest that variable isoform expression may play a role in this pleiotropic phenotype. In addition, we have created a new ld mutant that can be used to study factors involved in the penetrance of the renal agenesis phenotype.

\section{Renal Agenesis, but no Limb Phenotype, in Isoform IV-Deficient Mice}

We have demonstrated that the disruption of a specific isoform of the ld locus can isolate the limb and kidney phenotypes of a complex dysmorphic syndrome in mice. The loss of function of isoform IV is sufficient for the renal agenesis phenotype of the $l d$ locus, albeit at a low penetrance, providing compelling evidence for a role for this isoform and the $165-\mathrm{kDa}$ protein that it encodes in kidney development. We have also definitively shown that this gene is involved in the $l d$ phenotype, since the targeted disruption of isoform IV did not complement the renal agenesis phenotype of two other ld alleles. The noncomplementation of the kidney phenotype between $l d^{G K O}$ and $l d^{l n 2}$ is particularly compelling in this respect. However, isoform IV-deficient mice 
have normal limbs, demonstrating that the loss of this isoform is not sufficient for the ld limb phenotype. One potential explanation for the segregation of the limb and kidney phenotypes is that the $l d^{G K O}$ allele is weaker than the other known ld alleles; those mutant alleles in which the mutations have been characterized have disruptions of all of the known isoforms, whereas the isoform IV targeted allele specifically disrupts only this isoform, leaving intact the expression of isoforms I-III. This explanation is supported by the low penetrance of the renal phenotype in the isoform IV deficient mice. However, this cannot be a complete explanation. Although we do not know the molecular lesions of the $l d^{J}$ or $l d^{O R}$ alleles, the $l d^{J}$ and $l d^{O R}$ alleles have no mutations in isoform IV and hence do not disrupt all $l d$ isoforms, either. It is also possible that the molecular actions of the $l d$ gene products are distinct in the developing limb and kidney and that the pleiotropic phenotype is the result of variable isoform expression. This is supported by the different spatial and temporal patterns of expression of the RNA and protein isoforms of $l d$.

The developing AER is defective in ld mutants with limb abnormalities (25), which suggests that the origin of the limb defects in $l d$ mutants resides in the AER. Consequently, the expression pattern of $l d$ isoforms was examined, with the goal of identifying those isoforms which, by virtue of their expression in the AER, might contribute to the $l d$ limb phenotype. Isoform IV is expressed at high levels in the AER of developing limb buds $(11,12)$, yet no limb phenotype was observed in the isoform IV-deficient mice, suggesting that additional isoforms (and the proteins they encode) must complement a deficiency of isoform IV in the limb. Axial patterning in the developing limb bud is a result of reciprocal inductive interactions between the mesenchyme and ectoderm (for a recent review, see ref. 26). Molecularly, these interactions appear to result from a feedback loop in the mesenchyme and ectoderm, consisting of $S h h, f g f-4$, and Wnt-7a (27-34). This feedback loop is disrupted in $l d$ mutants (12). Thus, it is possible that defects in the AER of $l d$ mutants could result from primary abnormalities in the AER or mesenchyme. In support of the importance of the mesenchyme in the ld phenotype, wild-type mesenchyme can rescue the AER defect of $l d$ ectoderm in chimeric mouse/avian limbs (35). Consequently, isoforms that are expressed in the mesenchyme may play an important role in the ld phenotype. Taken together, these experiments suggest that an $l d$ isoform that is expressed anywhere in the developing limb could potentially be involved in the mutant limb phenotype. For example, low levels of RNA of isoforms I and II are present in the limb bud (10) and contain the $\mathrm{COOH}$-terminal region of the $l d$ gene disrupted in the $l d^{T g H d}, l d^{T g B r i}$, or $l d^{l n 2}$ alleles. These isoforms are unaltered in the isoform IV-deficient mice. Other candidate isoforms have been identified in embryonic limb bud ectoderm (11).

It is also clear that missense mutations of isoform IV are not necessary for either the limb or renal phenotypes of $l d$, since sequence analysis of two alleles $\left(l d^{J}\right.$ and $\left.l d^{O R}\right)$ failed to detect physiologically significant alterations in isoform IV protein structure. Both of these alleles have fully penetrant limb defects and highly penetrant renal agenesis. For example, the $l d^{J}$ allele has $98 \%$ penetrance, while the $l d^{O R}$ allele has $70 \%$ penetrance of renal agenesis (10). All of these results are best explained by a model in which isoforms other than isoform IV are necessary and sufficient for the limb phenotype of $l d$, whereas mutations in isoform IV are sufficient, but not necessary, for the renal agenesis phenotype.

The formation of the kidneys is a result of a reciprocal inductive interaction between the ureteric bud and metanephric blastema. At about E11 in the mouse, the ureteric bud grows out from the mesonephric duct into the metanephric blastema, resulting in the induction of the blastema to form kidney parenchyma. The differentiating blastema mesenchyme in turn signals the ureteric bud to continue to grow and branch. Renal agenesis can result from defects in either the ureteric bud or metanephric blastema. Delays in ureteric bud outgrowth appear to be the primary defect in $l d$ mutant homozygotes, leading to renal agenesis (10). We have previously shown that isoforms I-III and isoform IV are expressed in the pronephros, mesonephric tubules and ducts, the ureteric bud of the developing metanephros, and ureteric bud derivatives of the developing kidney by whole-mount in situ hybridization analysis (12). Thus, the ld isoforms, including isoform IV, are expressed in the developing kidney in a pattern consistent with an important role in ureteric bud outgrowth.

The phenotype of the isoform IV-deficient mice demonstrates that isoform IV is necessary for normal renal development and implies that this isoform is involved in ureteric bud outgrowth. In situ hybridization analysis of the $l d$ isoforms showed that isoform IV was not expressed in the ureteric bud of isoform IV-defi- 
cient mice, but isoform I-III expression was expressed normally. Although the presence of all other $l d$ isoforms is not sufficient for completely normal renal development, we have not ruled out roles for other $l d$ isoforms in renal development. Isoforms I-III may also be important for the normal development of the kidneys, a hypothesis that is supported by their pattern of expression in the embryo. It is possible that the other isoforms compensate somewhat, but not completely, for the loss of isoform IV in the $l d^{G K O} / l d^{G K O}$ mice.

\section{Penetrance and Renal Agenesis}

The renal agenesis phenotype of all $l d$ mutations displays incomplete penetrance and variable expressivity (10). In the isoform IV-deficient mice, this phenotype may have reduced penetrance relative to other $l d$ alleles because the $l d^{G K O}$ allele is a weaker allele, as discussed above, or because of strain differences in penetrance. This low penetrance is nevertheless consistent with the renal agenesis phenotype of other $l d$ mutants, where the penetrance ranges from $21 \%$ to $96 \%$ (10). The difference in penetrance among other $l d$ alleles may be due to differences in the rate of ureteric bud outgrowth, or to the sensitivity of particular strains to variations in the rate of outgrowth. For example, the $l d^{J}$ allele, with the highest penetrance, may result in a uniform delay in the rate of ureteric bud outgrowth such that nearly every animal has renal defects, while the $l d^{G K O}$ allele, with relatively low penetrance, may have a less severe effect on the rate of ureteric bud outgrowth. It has not been possible to test directly whether ureteric bud outgrowth is delayed in the isoform IV-deficient mice because of the low penetrance of renal agenesis. Complicating this analysis is the fact that all alleles of $l d$ arise and are maintained in different strain backgrounds. We are using these differences in penetrance to determine the role of individual alleles and strain-specific modifying alleles in determining the penetrance of renal agenesis in ld mutants.

Disruptions of other genes in mice have resulted in completely penetrant, recessive renal agenesis/dysgenesis: the Wilms tumor-associated transcription factor WT-1 (36); the c-ret receptor tyrosine kinase (37); the c-ret ligand GDNF (38-40); the secreted signaling molecule Wnt-4 (41), and the transcription factor Pax-2 (42). WT-1 $(43,44)$ and Wnt-4 $(41)$ are expressed in the mesenchymal condensations surrounding the growing ureteric bud and are likely involved in receiving the inductive signal in the metanephric blastema from the ureteric bud. Interference with mesenchymal induction in these mutants would be expected to result in completely penetrant renal agenesis. c-ret is expressed in the ureteric bud (45), and the elimination of this gene or its ligand GDNF must result in a more severe disruption of ureteric bud function than the described mutations of $l d$, since they result in $100 \%$ penetrant phenotypes. Pax-2 is expressed in both the epithelial and mesenchymal compartments of the developing kidney, and its loss also results in the loss of genital tracts, suggesting that Pax-2 is central to development of mesonephric as well as metanephric derivatives. Id mutants have defects in ureteric bud outgrowth only, but not in the later development of the kidney, since careful examination of the normalappearing kidneys in $l d$ mutant animals have revealed no abnormalities (G. Ryan and A. Wynshaw-Boris, unpublished observations). Thus, the defects in renal development in ld mutants most likely precede ureteric bud outgrowth and probably occur in the mesonephric ducts. We are currently examining the expression patterns of markers for mesonephric ducts and ureteric buds in $l d^{J} / l d^{J}$ mutants to address these issues.

Renal agenesis is also incompletely penetrant and variably expressed in humans, although it is unclear whether it is inherited in an autosomaldominant and/or autosomal-recessive manner $(46-48)$. By isolating the renal phenotype of $l d$ from the limb abnormalities, the isoform IVdeficient mouse provides a model for human renal agenesis and suggests a candidate gene for mutation examination.

\section{ACKNOWLEDGMENTS}

We thank En Li and Rudolph Jaenisch for providing the Jl ES cells, Cathie Daugherty and Anne Harrington for excellent technical assistance, and Lee Niswander for sharing unpublished data. We are grateful to Les Biesecker, Bill Pavan, and members of the Leder and Wynshaw-Boris labs for useful discussions and comments on the manuscript. A. W.-B. was a Howard Hughes Medical Institute Physician Research Fellow. 


\section{REFERENCES}

1. Lyon MF, Searle AG. (1989) Genetic Variants and Strains of the Laboratory Mouse, 2nd ed. Oxford University Press, Oxford.

2. Jones KL. (1988) Smith's Recognizable Patterns of Human Malformation. W.B. Saunders, Philadelphia.

3. Cupp MB. (1960) Mouse News Letter 22:50.

4. Cupp MB. (1962) Mouse News Letter 26:51.

5. Green MC. (1962) Mouse News Letter 26:34.

6. Kleinebrecht J. (1974) Mouse News Letter 51: 19.

7. Woychik RP, Stewart TA, Davis LG, D'Eustachio P, Leder P. (1985) An inherited limb deformity created by insertional mutagenesis in a transgenic mouse. Nature 318: 36-40.

8. Messing A, Behringer RR, Slapeak JR, Lemke G, Palmiter RD, Brinster RL. (1990) Insertional mutagenesis at the ld locus (again!) in a line of transgenic mice. Mouse Genome 87:107.

9. Woychik RP, Generoso WM, Russell LB, Cain KT, Cacheiro NL, Bultman SJ, Selby PB, Dickinson ME, Hogan BL, Rutledge JC. (1990) Molecular and genetic characterization of a radiation-induced structural rearrangement in mouse chromosome 2 causing mutations at the limb deformity and agouti loci. Proc. Natl. Acad. Sci. U.S.A. 87:25882592.

10. Maas R, Elfering S, Glaser T, Jepeal L. (1994) Deficient outgrowth of the ureteric bud underlies the renal agenesis phenotype in mice manifesting the limb deformity (ld) mutation. Dev. Dyn. 199:214-228.

11. Jackson-Grusby L, Kuo A, Leder P. (1992) A variant limb deformity transcript expressed in the embryonic mouse limb defines a novel formin. Genes Dev. 6:29-37.

1la.Wang CC, Chan DC, Leder P (1997) The mouse formin $(F m n)$ gene: genomic structure, novel exons, and genetic mapping. Genomics 39:303-311.

12. Chan DC, Wynshaw-Boris A, Leder $P$. (1995) Formin isoforms are differentially expressed in the mouse embryo and are required for normal expression of $f g f-4$ and $s h h$ in the limb bud. Development 121:1351-1362.

13. Trumpp A, Blundell PA, de la Pompa J, Zeller R. (1992) The chicken limb deformity gene encodes nuclear proteins expressed in specific cell types during morphogenesis. Genes Dev. 6: 14-28.
14. Tybulewicz VL, Crawford CE, Jackson PK, Bronson RT, Mulligan RC. (1991) Neonatal lethality and lymphopenia in mice with a homozygous disruption of the c-abl protooncogene. Cell 65:1153-1163.

15. Li E, Bestor TH, Jaenisch R. (1992) Targeted mutation of the DNA methyltransferase gene results in embryonic lethality. Cell 69: 915-926.

16. Deng C-X, Wynshaw-Boris A, Shen MM, Daugherty C, Ornitz D, Leder P. (1994) Murine FGFR-1 is required for early postimplantation growth and axial organization. Genes Dev. 8:3045-3057.

17. McLeod MJ. (1980) Differential staining of cartilage and bone in whole mouse fetuses by alcian blue and alizarin red S. Teratology 22: 299-301.

18. Kleinebrecht J, Selow J, Winkler W. (1982) The mouse mutant limb-deformity (ld). Anat. Anz. 152:313-324.

19. Vogt TF, Jackson-Grusby L, Wynshaw-Boris AJ, Chan DC, Leder P. (1992) The same genomic region is disrupted in two transgene-induced limb deformity alleles. Mamm. Genome 3:431-437.

20. Wilkinson DG. (1992) Whole mount in situ hybridization of vertebrate embryos. In: Wilkinson DG (ed). In Situ Hybridization: $A$ Practical Approach. IRL Press, Oxford, pp. 7583.

21. Rosen B, Beddington RSP. (1993) Wholemount in situ hybridization in the mouse embryo: Gene expression in three dimensions. Trends Genet. 9:162-167.

22. Harlow E, Lane D. (1988) Antibodies: A Laboratory Manual. Cold Spring Harbor Laboratory Press, Cold Spring Harbor, New York.

23. Woychik RP, Maas RL, Zeller R, Vogt TF, Leder P. (1990) 'Formins': Proteins deduced from the alternative transcripts of the limb deformity gene. Nature 346:850-853.

24. Vogt TF, Jackson-Grusby L, Rush J, Leder P. (1993) Formins: phosphoprotein isoforms encoded by the mouse limb deformity locus. Proc. Natl. Acad. Sci. U.S.A. 90:5554-5558.

25. Zeller R, Jackson-Grusby L, Leder P. (1989) The limb deformity gene is required for apical ectodermal ridge differentiation and anteroposterior limb pattern formation. Genes Dev. 3:1481-1492.

26. Tabin C. (1995) The initiation of the limb bud: Growth factors, Hox genes, and retinoids. Cell 80:671-674.

27. Riddle RD, Johnson RL, Laufer E, Tabin C. 
(1993) Sonic hedgehog mediates the polarizing activity of the ZPA. Cell 75:1401-1416.

28. Johnson RL, Riddle RD, Laufer E, Tabin C. (1994) Sonic hedgehog: A key mediator of anterior-posterior patterning of the limb and dorso-ventral patterning of axial embryonic structures. Biochem. Soc. Trans. 22:569-574.

29. Niswander L, Martin GR. (1993) Mixed signals from the AER: FGF-4 and Bmp-2 have opposite effects on limb growth. Prog. Clin. Biol. Res. 383B:625-633.

30. Niswander L, Tickle C, Vogel A, Booth I, Martin GR. (1993) FGF-4 replaces the apical ectodermal ridge and directs outgrowth and patterning of the limb. Cell 75:579-587.

31. Niswander L, Martin GR. (1993) FGF-4 and BMP-2 have opposite effects on limb growth. Nature 361:68-71.

32. Niswander L, Tickle C, Vogel A, Martin G. (1994) Function of FGF-4 in limb development. Mol. Reprod. Dev. 39:83-88; discussion $88-89$.

33. Niswander L, Jeffrey S, Martin GR, Tickle C. (1994) A positive feedback loop coordinates growth and patterning in the vertebrate limb. Nature 371:609-612.

34. Parr BA, McMahon AP. (1995) Dorsalizing signal Wnt-7a required for normal polarity of D-V and A-P axes of mouse limb. Nature 374:350-353.

35. Kuhlman J, Niswander L. (1997) Limb deformity proteins: Role in mesodermal induction of the apical ectodermal ridge. Develop ment 124:133-139.

36. Kreidberg JA, Sariola H, Loring JM, Maeda $M$, Pelletier J, Housman D, Jaenisch R. (1993) WT-1 is required for early kidney development. Cell 74:679-691.

37. Schuchardt A, D'Agati V, Larsson-Blomberg L, Costantini F, Pachnis V. (1994) Defects in the kidney and enteric nervous system of mice lacking the tyrosine kinase receptor Ret. Nature 367:380-383.

38. Sanchez MP, Silos-Santiago I, Frisen J, He B, Lira SA, Barbacid MA. (1996) Renal agenesis and the absence of enteric neurons in mice lacking GDNF. Nature 382:70-73.
39. Pichel JG, Shen L, Sheng HZ, Granholm A-C, Drago J, Grinberg A, Lee EJ, Huang SP, Saarma M, Hoffer BJ, Sariola H, Westphal H. (1996) Defects in enteric innervation and kidney development in mice lacking GDNF. Nature 382:73-76.

40. Moore MW, Klein RD, Farinas I, Sauer H, Armanini M, Phillips H, Reichardt LF, Ryan AM, Carver-Moore K, Rosenthal A. (1996) Renal and neuronal abnormalities in mice lacking GDNF. Nature 382:76-79.

41. Stark K, Vainio S, Vassileva G, McMahon A. (1994) Epithelial transformation of metanephric mesenchyme in the developing kidney regulated by Wnt-4. Nature 372:679683.

42. Torres M, Gomez-Pardo E, Dressler GR, Gruss P. (1995) Pax-2 controls multiple steps of urogenital development. Development 121: 4057-4065.

43. Pelletier J, Schalling $M$, Buckler AJ, Rogers A, Haber DA, Housman D. (1991) Expression of the Wilm's tumor gene WT-1 in the murine urogenital system. Genes Dev. 5:1345-1356.

44. Armstrong JF, Pritchard-Jones $\mathrm{K}$, Bickmore WA, Hastie ND, Bard JBL. (1992) The expression of the Wilm's tumor gene, WT-1, in the developing mammalian embryo. Mech. Dev. 40:85-97.

45. Pachnis V, Mankoo BS, Costantini F. (1993) Expression of the c-ret proto-oncogene during mouse embryogenesis. Development 119: 1005-1017.

46. Roodhooft AM, Birnholz JC, Holmes LB. (1984) Familial nature of congenital absence and severe dysgenesis of both kidneys. New Engl. J. Med. 310:1341-1345.

47. Bankier A, deCampo $M$, Newell R, Rogers JG, Danks DM. (1985) A pedigree study of perinatally lethal renal disease. J. Med. Genet. 22:104-1 11 .

48. Battin J, Lacombe D, Leng J-J. (1993) Familial occurrence of hereditary renal adysplasia with Mullerian anomalies. Clin. Genet. 43: 23-24. 